\title{
Reducing the need for VCUG-a new proteomic screening tool for VUR
}

A German-led study has determined that several newly identified polypeptide biomarkers in urine can be used to diagnose high-grade vesicoureteral reflux (VUR), which is a common occurrence in children with UTI or hydronephrosis. The peptides can be identified using a practical, noninvasive test that might rival voiding cystourethrogram (VCUG), the current gold-standard procedure for diagnosing and determining the severity of the condition.

The researchers characterized the urinary proteome of VUR using specimens from 18 patients with confirmed grade IV or V primary VUR (in at least one kidney, with no prior surgical interventions) and 19 age-matched controls. Their analysisusing capillary electrophoresis and mass spectrometry-revealed reduced expression of nine polypeptides in patients with severe VUR. Interestingly, the proteomic pattern of these children was different to that of patients with ureteropelvic junction obstruction, highlighting a potential method for differentiating these two urologic manifestations using the analytical technique. Finally, the researchers built a diagnostic model of the proteomic data using the support vector machine (SVM) learning technique. SVM is a powerful tool for classifying data into two groups; either a given patient's urinary 'fingerprint' matches the VUR proteome-which would then qualify him or her for VCUG confirmation-or not.

Urine samples from 17 patients and 19 controls were then used in a blinded

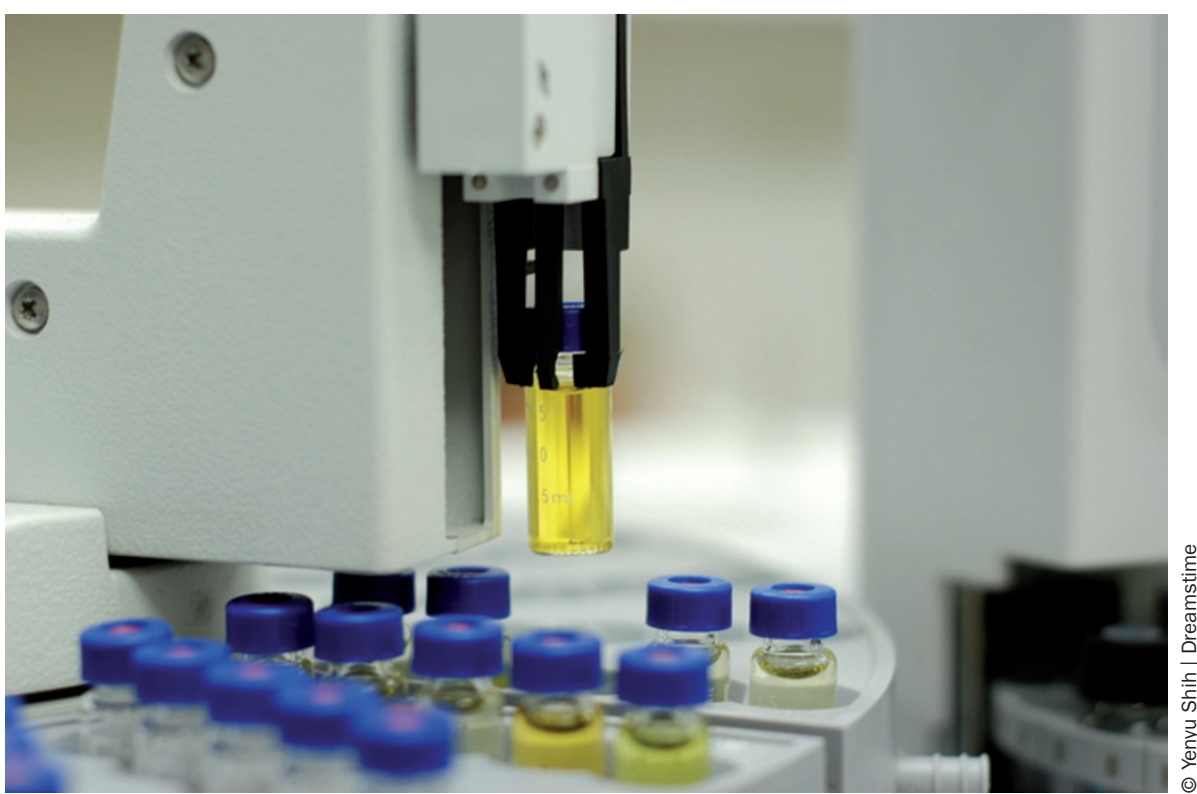

assessment of the technique. The SVM algorithm has specific values of sensitivity and specificity that can be selected by setting the cut-off value, empowering the user to decide how precise the tool is at screening for VUR. For example, an SVM cut-off value of -0.15 corresponded to an $88 \%$ sensitivity (95\% CI 64-98\%) and $79 \%$ specificity (95\% CI 54-94\%) for detecting high-grade VUR. At this level of precision, the odds ratio that a patient with a positive proteome test had high-grade VUR was 28. "The most significant result of our study is the reproducibility of the pattern in blinded analysis," investigator Jens Drube told Nature Reviews Urology. "The test can identify severe VUR in a patient cohort that is heterogeneous with respect to age and grade of reflux of the contralateral kidney."

The strengths of this analytical assessment are obvious: the authors estimate that more than $50 \%$ of unnecessary VCUGs can be avoided, saving young patients from radiation exposure and stress. Additionally, the high throughput analysis is fully automated and SVM results can be obtained within $48 \mathrm{~h}$ of urine collection. However, the small study size currently limits its strength. "Prompt validation of these promising biomarkers in a sufficiently powered prospective cohort [is needed] to make this new approach accessible to our young patients," Drube concluded.

Mina Razzak

Original article Drube, J. et al. Urinary proteome analysis to exclude severe vesicoureteral reflux. Pediatrics doi:10.1542/peds.2010-3467 